\title{
MiR-124 Attenuates Osteoclastogenic Differentiation of Bone Marrow Monocytes Via Targeting Rab27a
}

\author{
Lian Tang Yiran Yin Juncai Liu Zhong Li Xiaobo Lu \\ Department of Orthopaedics, the affiliated Hospital of Southwest Medical University, Luzhou City, \\ China
}

\section{Key Words}

MiR-124 • Rab27a • Bone Marrow Monocytes • Osteoclasts • Osteoporosis

\begin{abstract}
Background/Aims: With the aging population increases, senile osteoporosis has become a global public health problem. Previous evidence has shown that miR-124 has important effects on the occurrence and development of osteoporosis. However, the role of miR-124 in the process of osteoclastogenesis is still obscure. Methods: First of all, we measured the expression level of miR-124 in bone marrow monocytes (BMMs) of osteoporotic mice (ovariectomized mice: OVX). Next, we evaluated the alteration of miR-124 during osteoclast differentiation of BMMs. Then, BMMs were transfected with miR-124 mimics or inhibitors to explore the influences of miR-124 on osteoclast differentiation of BMMs in vitro. Furthermore, bioinformatics analysis and luciferase reporter assay were performed for prediction and identification of the target of miR-124. Results: BMMs from OVX mice exhibited lower expression of miR-124 compared with Sham mice. Additionally, miR-124 was down-regulated when BMMs differentiated into osteoclasts. In addition, inhibition of miR-124 promoted BMMs differentiated into osteoclasts in vitro, whereas overexpression of miR-124 attenuated this procedure, demonstrated by increased expression of osteoclast specific genes and TRAP staining. Furthermore, Rab27a was confirmed to be the direct target of miR-124 by bioinformatics, Western blot and luciferase reporter assay analysis. Conclusion: Our findings revealed that miR-124 has an important role in osteoclastogenesis via targeting Rab27a. Thus, targeting miR-124 promises a therapeutic potential in the treatment of osteoporosis.

(C) 2017 The Author(s)

Published by S. Karger AG, Basel
\end{abstract}

\section{Introduction}

Bone is a tissue that demands coordinated dynamic equilibrium of osteoclastic bone resorption and osteoblastic bone formation. Osteoporosis is carried out by excessive bone destruction by osteoclasts coupled with insufficient bone formation by osteoblasts, resulting in reduction of bone mass and low trauma fractures [1, 2]. 
MicroRNAs (miRNAs) are evolutionarily conserved, small ( $\sim 22$ nucleotides), singlestranded non-coding RNAs that silence the expression of specific gene by base-pairing to the 3'-untranslated regions of target mRNAs on post-transcriptional level [3-5]. At present, numerous studies have indicated that miRNA was a crucial component in the development of osteoporosis [6-11]. For example, miR-34a blocks osteoporosis by inhibiting Tgif2 [12]; miRNA-148a regulates osteoclastogenesis via targeting MAFB [13]; and miRNA-142-3p induces cell death in osteoclasts depending on RANKL [14]. Among, miR-124 was previously reported to be related to osteoporosis. However, whether miR-124 takes part in the occurrence and development of osteoporosis still remains unclear. In the present study, we aimed to elucidate the role of miR-124 played in osteoclast differentiation and to probe the potential molecular mechanism.

\section{Materials and Methods}

\section{Establishment of osteoporosis models}

All procedures involving mice were approved by the Animal Ethic Committee of Southwest Medical University. Female C57BL/6J mice obtained from Animal center of University were maintained in conditions with 12 hours of light, 12 hours of darkness and free access to food and water. The mice were randomly divided into ovariectomized $(n=3)(O V X)$ or sham-operated $(n=3)$ (Sham) groups when arrived the age of 6 months. After the operation, mice were maintained in normal condition for 3 months, then, performed with CT scanning (micro-CT, Siemens AG, Germany). We recorded several evaluation criteria for osteoporosis including bone mineral density (BMD), trabecular thickness (Tb.Th, $\mathrm{mm}$ ), bone volume relative to tissue volume (BV/TV, \%), and trabecular separation (Tb.Sp, mm). After the verification, we gathered bilateral femurs of OVX and Sham mice for further extraction of BMMs.

\section{Extraction and culture of BMMs}

Bone marrow-derived monocyte cells (BMMs) from the collected femurs were isolated as described previously $[15,16]$. In brief, the bone marrow cells isolated from mice bilateral femurs (OVX, Sham or Normal group) were cultured (1*105 cells/well; 96-well plate) in $\alpha$-MEM (Sigma) supplemented with $10 \%$ FBS (GIBCO). After incubating bone marrow cells with M-CSF (Sigma, USA) at the concentration of 20ng/ml for 2 days, adherent cells were served as bone marrow-derived monocyte (BMM).

\section{Osteoclastogenic differentiation of BMMs}

Using the BMMs harvested from normal mice (6-8 weeks old) to performing osteoclast differentiation experiments. BMMs were co-cultured with RANKL $(50 \mathrm{ng} / \mathrm{ml})$ containing M-CSF (20ng/ml) for different period to induce osteoclast differentiation. Osteoclasts induced in the culture were assessed by TRAP staining, using a commercial kit (Sigma Chemical). TRACP+ multinuclear osteoclasts ( $>3$ nuclei) appeared as dark purple cells and were counted by light microscopy.

\section{MiRNA mimic/inhibitor transfection}

MiR-124 mimics, miR-124 inhibitors, and a negative control (NC) with fluorescent tags were synthesized by Shanghai GenePharma (Shanghai, China). For transfection, BMMs were seeded in 12-well plates $(5 \times 104$ cells $/ \mathrm{ml})$ and transfected with $100 \mathrm{nM}$ of miR-124 mimics, $150 \mathrm{nM}$ of miR-124 inhibitors or NC using lipo-3000 (Life Technologies Corporation, Gaithersburg, MD, USA). After being incubated for 24 hours, the transfection efficiency was observed under fluorescence microscope. Then, the cells were cultured for subsequent experimental research with normal medium.

\section{Cell viability and proliferation}

The BMMs were inoculated on a six-well plate made by flexible silicone rubber at a density of $1.0 \times 104$ cells per well. After maintained for 12 hours at the temperature of $37^{\circ} \mathrm{C}$ with $5 \% \mathrm{CO} 2$, the cells were treated with the mimics or miR-124 inhibitors respectively before performing the cell proliferation assay.

After transfection, Alamar Blue reagent (10\% of the sample volume; Invitrogen, USA) was added at $0,24,48$, and 72 hours respectively. After incubating in the dark for 4 hours, the absorbance of $100 \mathrm{uL}$ 


\section{Cellular Physiology Cell Physiol Biochem 2017;43:1663-1672 \begin{tabular}{l|l|l} 
DOI: 10.1159/000484027 & $\begin{array}{l}\text { C } 2017 \text { The Author(s). Published by S. Karger AG, Basel } \\
\text { www.karger.com/cpb }\end{array}$
\end{tabular}}

Yin et al.: Role of MiR-124 in Osteoclastogenesis

medium separated into a 96-well plate at $570 \mathrm{~nm}$ and $590 \mathrm{~nm}$ was calculated using a spectrophotometer (TECAN, Switzerland). An empty plate without cells served as blank group. Cells transfected with negative control served as control group. All values were subjected to three separate experiments, and the results were recorded for analysis based on the machine description.

\section{RNA isolation}

Total RNA was extracted from established cells by using Trizol (Ambion, Austin, TX, USA). In short, the cells were gathered in an EP tube, lysed with $1 \mathrm{ml} / 10 \mathrm{~cm}^{2}$ Trizol and then mixed with chloroform $(0.2 \mathrm{ml} / \mathrm{ml}$ Trizol). After centrifuging the EP tube at $12,000 \mathrm{rpm}$ for $20 \mathrm{~min}\left(\right.$ at $\left.4^{\circ} \mathrm{C}\right)$, the liquid supernatant was removed to a fresh EP tube and mixed with cold isopropanol $(0.5 \mathrm{ml} / \mathrm{ml}$ Trizol). The mixture was centrifuged at same condition described before for $10 \mathrm{~min}$, then the upper liquid was discarded and the RNA precipitation was obtained. The RNA precipitation was washed twice in $75 \%$ ethanol, then dried at room temperature naturally, and then each tube added 20 50ul DEPC water to dissolve the RNA precipitation. After the detection of the concentration, the RNA solution was prepared for the next analysis.

\section{qRT-PCR analysis}

A PrimeScript ${ }^{\mathrm{TM}}$ RT reagent kit (TaKaRa, Tokyo, Japan) was used to reverse transcript the RNA to cDNA. Then, real-time RT-PCR was conducted using the SYBR Premix Ex Taq II kit (TaKaRa, Tokyo, Japan) and small nuclear RNA U6 was used as internal control. All samples were tested three times independently and the relative expression levels were calculated using the 2- $\Delta \Delta \mathrm{CT}$ method. Specific primers for mouse miR-124 and U6 were ordered from Sangon Biotech. The sequences were displayed as follows. U6 (forward: 5'-AGAGAAGATTAGCATGGCCCCTG-3', reverse: 5'-ATCCAGTGCAGGGTCCGAGG-3'); miR-124 (forward: 5'-CGGTGATTCCGTGCGCCACT-3', reverse: 5'-ATCCAGTGCAGGGTCCGAGG-3'); NFATc1 (forward: 5'-AAGAGGAAGTACAGCCTCAACG-3', reverse:5'-TCTCCTTTCCGAAGTTCAATGT-3'); TRAP (forward: 5'-GGAGGGAATAAAGGCTCAGG-3', reverse: 5'-GGAACTCAGCAAAGGTGAGC-3'); Rab27a (forward: 5'-AGGGCAGTGAAAGAGGAGGA-3', reverse: 5'-TCCAGGAGCATCTCAATCGC-3'); $\beta$-actin (forward: 5'-TCCTGTGGCATCCACGAAACT-3', reverse: 5'-GAAGCATTTGCGGTGGACGAT-3').

\section{Target gene prediction}

TargetScan (http://www.targetscan.org/), miRanda (http://www.microrna.org/microrna/) and PicTar (http://pictarmdc-berlin.de/) databases were used to predict the potential targets of miR-124. Rab27a was found to have a miR-124 binding site which located in its 3'-UTR region among the candidate target genes correlated with the osteoclast activity which had important effects in osteoclastogenic differentiation.

\section{Dual-luciferase reporter assay}

The dual-luciferase reporter system of Rab27a 3'-UTR was ordered from Genechem (Shanghai, China), and wild type and mutant Rab27a 3'-UTR were synthesized. To detect repression by miR-124, HEK293T cells were co-transfected with the indicated Rab27a 3'-UTR luciferase reporter (with wild-type or mutant miR124 binding regions). The Renilla luciferase was transfected as an efficiency control. The luciferase activity was measured using luciferase assay system (Promega, Madison, WI, USA) according to the instructions of the manufacturers.

\section{Western blot analysis}

RIPA (Beyotime, Shanghai, China) was used to extract the protein from experiment cells and concentration of total protein was measured by using a BCA assay kit (Beyotime, Shanghai, China) according to the guidance of the manufacturers. Total protein $(50 \mu \mathrm{g})$ was boiled with $5 \times$ loading buffer for $5 \mathrm{~min}$, and loaded and resolved on 8-12\% SDS-PAGE after cooling down. Then, the proteins separated were shifted to a PVDF membrane (Bio-Rad, Hercules, CA, USA). The membranes were immersed in 5\% BSA dissolved in TBST buffer for $1 \mathrm{~h}$ at $4^{\circ} \mathrm{C}$ and then incubated overnight with specific primary antibody at $4^{\circ} \mathrm{C}$. Rabbit monoclonal Trap antibody (dilution: 1/500; CatNOs: ab133238); rabbit polyclonal NFATc1 antibody (dilution: 1/200; CatNOs: ab25916) and rabbit polyclonal Rab27a antibody (dilution: 1/200; CatNOs: ab124325) were all purchased from Abcam (Cambridge, MA, USA). The membranes were incubated with secondary goat antirabbit (HRP) IgG antibody (dilution: 1/2000; CatNOs: ab6721; Abcam, Cambridge, MA, USA) for $2 \mathrm{~h}$ at room 


\section{Cellular Physiology Cell Physiol Biochem 2017;43:1663-1672

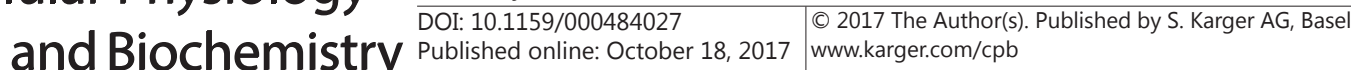

Yin et al.: Role of MiR-124 in Osteoclastogenesis

temperature after removing unbound primary antibody, and then washed with TBST buffer three times. The membranes were detected using an ECL kit (Millipore, Billerica, MA, USA). Three independent experiments were completed before data statistics.

\section{TRAP staining of BMMS}

Tartrate resistant acid phosphatase (TRAP) staining was conducted to confirm the osteoclasts according to the manufacturer's instructions (Sigma, St. Louis, MO, USA). Briefly, cells were fixed with stationary liquid for $30 \mathrm{~s}$ at room temperature and then washed with $\mathrm{dH} 2 \mathrm{O}$ three times. To prepare the staining solution, added the following reagent in a $100 \mathrm{ml}$ beaker: $45 \mathrm{ml} \mathrm{dH} 20\left(37^{\circ} \mathrm{C}\right), 2 \mathrm{ml}$ mixture fluid ( $1 \mathrm{ml}$ six azo pararosaniline liquid and $1 \mathrm{ml}$ acid salt solution) , $1 \mathrm{ml}$ tartaric acid salt solution and $0.5 \mathrm{~g}$ naphthalene cool AS-BI phosphate. Cells were immersed in the staining solution for $1 \mathrm{~h}$ at $37^{\circ} \mathrm{C}$, followed by washing with $\mathrm{dH} 2 \mathrm{O}$ three times. After that the cells were counterstained with hematoxylin for $90 \mathrm{~s}$ and then washed with alkaline solution for several minutes. Cells stained red and contained more than 3 nuclei were defined as TRAP-positive cells.

\section{Statistical analysis}

Data were expressed as the mean \pm SD and were analyzed with Student's T-test using SPSS version 16.0 software (IBM, NY, USA). Differences among groups were tested by one-way ANOVA. Statistical analyses between two groups were evaluated based on the Student's two-tailed t- test. Differences between groups were evaluated using Fisher's least-significant difference post-test procedure. Differences were considered statistically significant when the p value was under 0.05 .

\section{Results}

MiR-124 level down-regulated in osteoporotic mice BMMs

Both OVX and Sham mice were scanned using micro-CT to prove that OVX-mice matched to osteoporosis, and the indexes BMD, BV/TV, Tb.Th and Tb.Sp were recorded (Fig. 1A). Then BMMs were and distinguished with Flow cytometry. To study the difference of miR-124 expression in OVX-BMMs and Sham-BMMs, the qRT-PCR was conducted. The results revealed that miR-124 expressed significantly lower in OVX-BMMs than Sham-BMMs $(\mathrm{p}<0.05)$ (Fig. 1B).

\section{MiR-124 expressed lower level during osteoclast differentiation}

BMMs isolated from normal mice were treated with RANKL $(50 \mathrm{ng} / \mathrm{ml})$ and M-CSF $(20 \mathrm{ng} / \mathrm{ml})$ for different period ( 1 day, 3 day and 6 day) to induce osteoclast differentiation. Osteoclast formation was identified by increased expression of osteoclastogenesis-related genes (NFATc1 and TRAP) (Fig. 2A) and enhance TRAP staining (Fig. 2B). Further, qRTPCR was conducted at days 1,3 , and 6 to explore the change of miR-124 during osteoclast differentiation of BMMs. As expected, results revealed a significant decrease of miR-124 in BMMs after being differentiated into osteoclasts (Fig. 2C).

MiR-124 has no effect on cell viability of BMMs

Alamar Blue assay was conducted to probe the effects of miR-124 on the BMMs' viability. Cells treated with miR-124 mimics or miR-124 inhibitors revealed no significant change in cell viability, which suggested that up-regulation or down-regulation of miR-124 had no marked effects on BMMs proliferation (Fig. 3).

\section{MiR-124 inhibited osteoclast differentiation of BMMs}

To study the possible influence of miR-124 in the program of BMMs osteoclast differentiation, cells transfected with miR-124 mimics or miR-124 inhibitors were induced to differentiate into osteoclasts. Transfection efficiency was observed under fluorescence microscope and further demonstrated by qRT-PCR of miR-124 (Fig. 4A and B). At day 6 after osteoclastogenic induction using RANKL and M-CSF, qRT-PCR and western blot assay were 
Fig. 1. MiR-

124 was low expressed in BMMs isolated from OVX mice. A: micro-CT images for Sham and OVX mice. Bone parameters were calculated based on micro-CT images. BMD, BV/ $\mathrm{TV}$, and Tb.Th were lower in OVX-mice, and Tb.Sp was higher in OVXmice, compared with Sham mice ( ${ }^{*}$ C o m pared with Sham
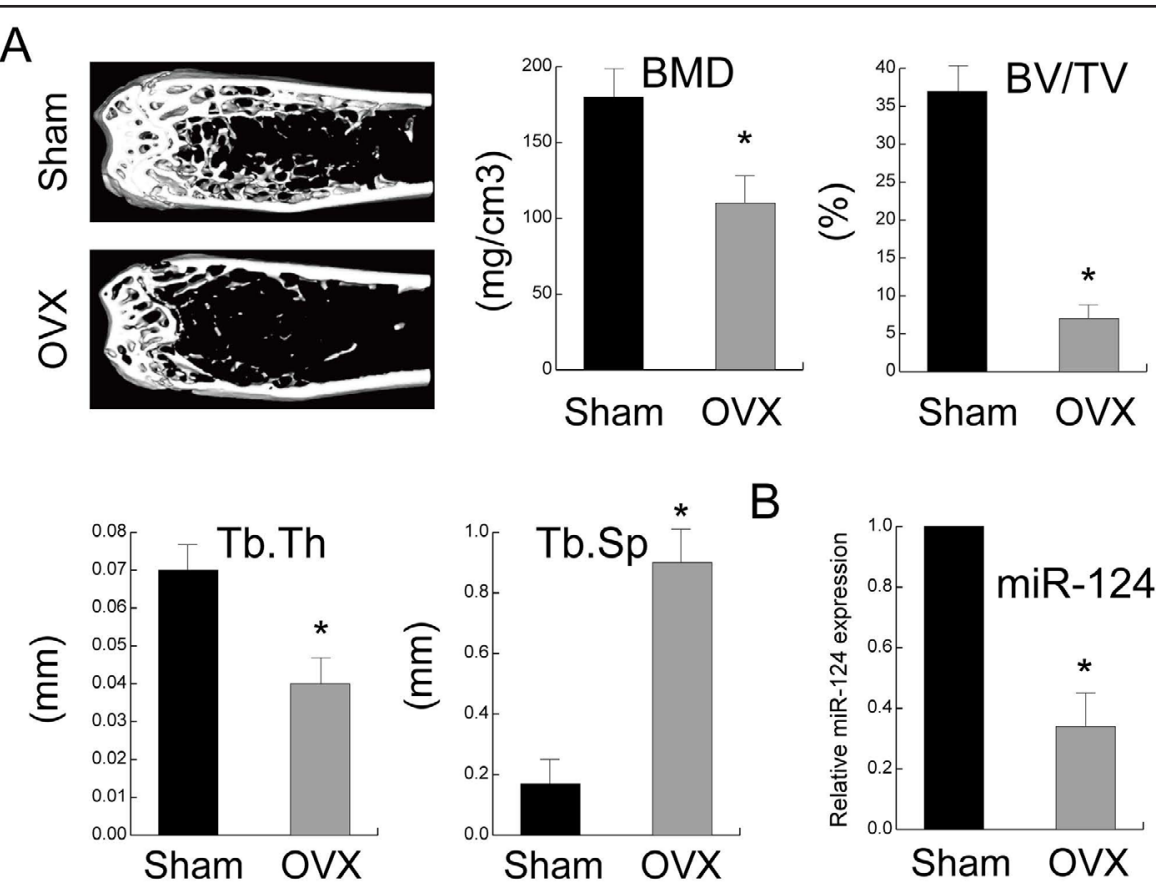

$B$

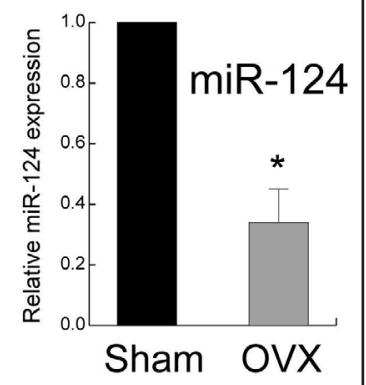

group, $\mathrm{p}<0.05$ );

B: qRT-PCR was performed to measure miR-124 expression in BMMs from Sham and OVX mice. Compared with Sham-mice, miR-124 was significantly lower in BMMs of OVX-mice (*Compared with Sham group, p <0.05). (BMD: bone mineral density; BV/TV: trabecular bone volume per tissue volume; Tb.Th: trabecular thickness; Tb.Sp: trabecular separation).

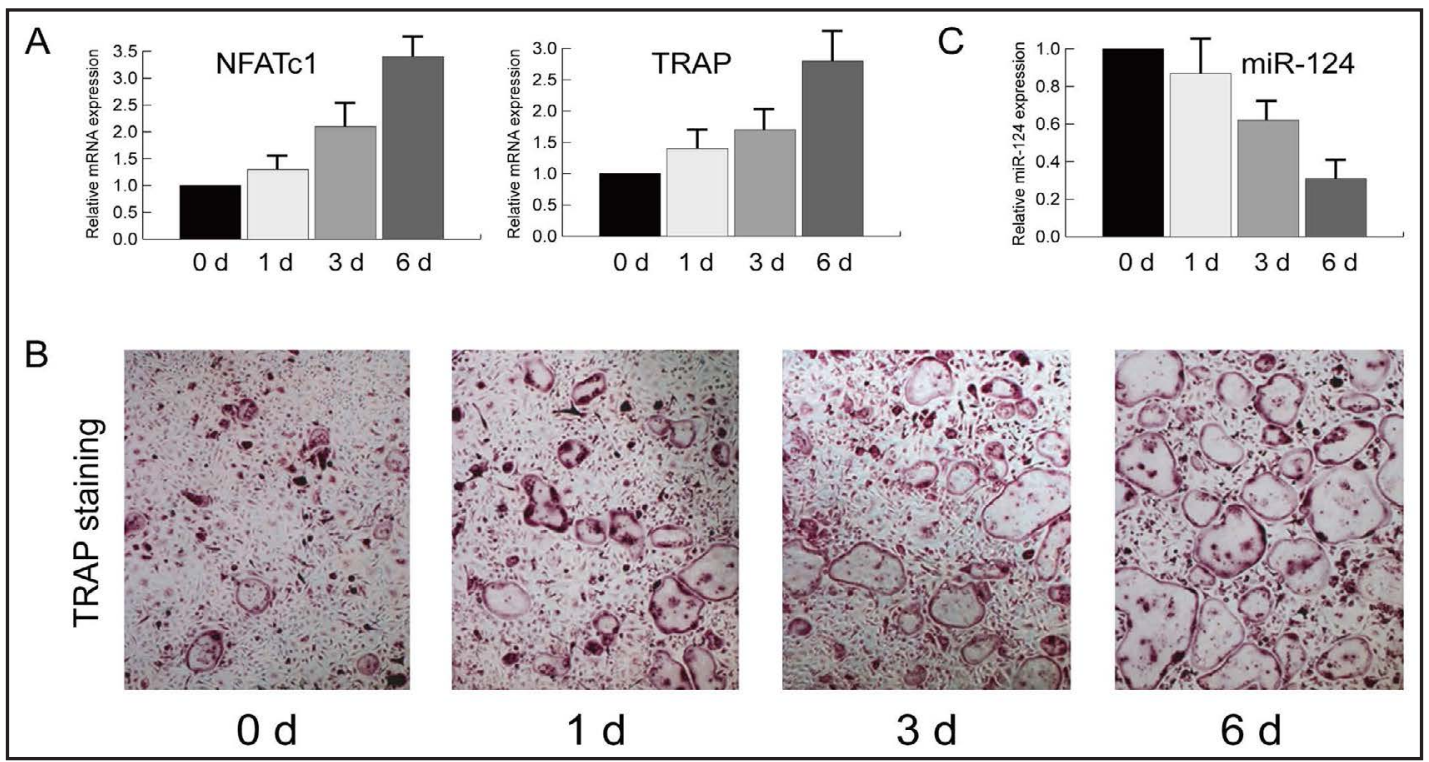

Fig. 2. MiR-124 was down-regulated during osteoclast differentiation. A: Two osteoclastogenesis-associated genes, NFATc1 and TRAP, were explored as evidence for osteoclastogesis. B: Trap staining was performed to evaluate the formation of multinucleated cells; C: BMMs treated with RANKL and C-MSF exhibited decreased miR-124 expression.

performed to explore expression profiles of NFATc 1 and TRAP in 3 different groups (miR124 mimics, miR-124 inhibitors and NC) (Fig. 4C and D). In addition, TRAP staining was also 
Fig. 3. The effects of miR-124 on cell proliferation were evaluated by Alamar Blue assay. BMMs transfected with miR-124 mimics or miR-124 inhibitors did not contributed to alteration of cell viability at $24 \mathrm{~h}, 48 \mathrm{~h}$ or $72 \mathrm{~h}$.
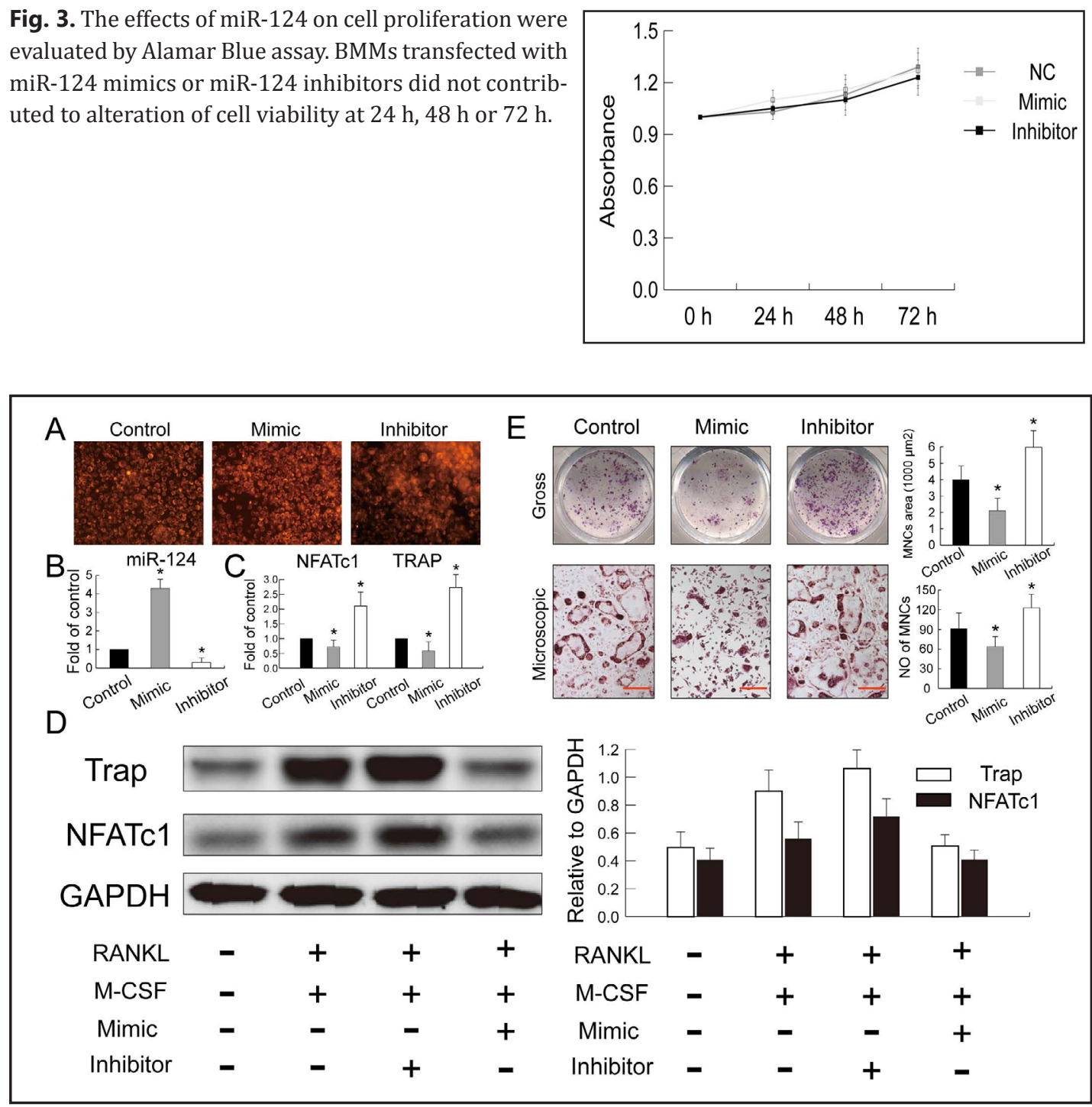

Fig. 4. MiR-124 inhibited osteoclastogenesis of BMMs. A: Transfection efficiency was observed under fluorescence microscope B: and further demonstrated by qRT-PCR of miR-124. Mimics infection induced more than 4 fold of elevation on miR-124 $\left({ }^{*}\right.$ Compared with control group, $\left.\mathrm{p}<0.05\right)$. C: Inhibition of miR-124 remarkably increased mRNA expression of NFATc1 and TRAP ( ${ }^{*}$ Compared with control group, p<0.05); D: Inhibition of miR-124 significantly elevated protein levels of NFATc1 and TRAP $(\mathrm{P}<0.05)$; E: Gross and microscopic images for Trap staining. Transfection with miR-124 inhibitors resulted in a significantly enhanced staining. Both the average size in areas and the number of TRAP-positive multinucleated OCs ( $>3$ nuclei) in miR-124 inhibitor group were more than those in other groups $\left({ }^{*}\right.$ Compared with control group, $\left.\mathrm{p}<0.05\right)$.

conducted at day 6 to detect osteoclast differentiation (Fig. 4E). Results showed that downregulation of miR-124 remarkably stimulated osteoclastic differentiation, demonstrated by increased expression of the osteoclast-specific genes NFATc1 and TRAP and increased TRAP staining. However, overexpression of miR-124 significantly inhibited osteoclastic differentiation of BMMs $(p<0.05)$.

\section{MiR-124 direct targeted Rab27a to effect BMMs osteoclast differentiation}

In order to further explore the potential mechanism involved in the regulation of miR124 in BMMs osteoclast differentiation, we searched the programs of TargetScan, PicTar and miRanda to detect the potential genes with a function of stimulating osteoclastogenesis. 


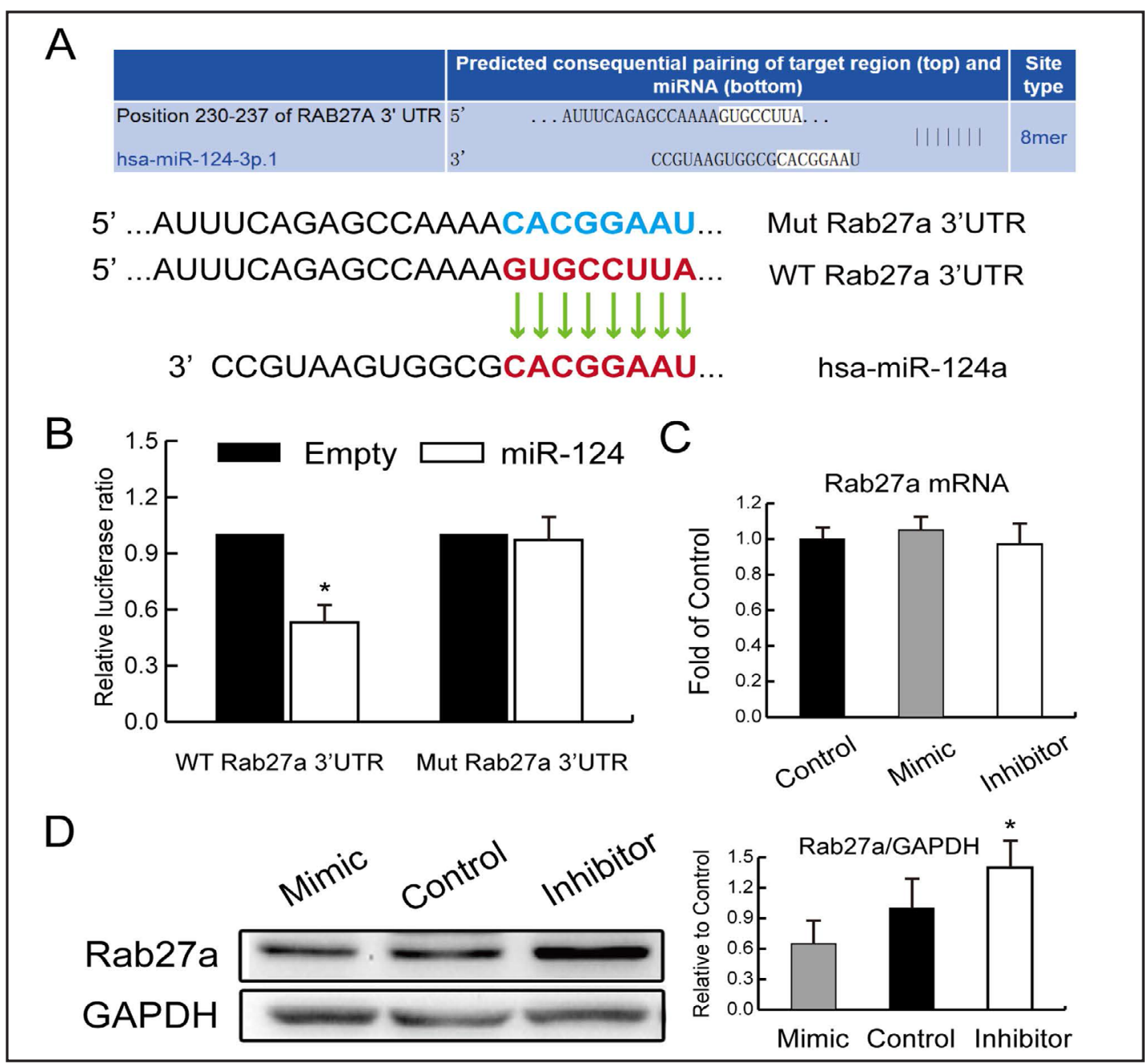

Fig. 5. Rab27a was a direct target of miR-124. A: Schematic diagram illustrating the design of luciferase reporters with the wild-type Rab27a 3'UTR (WT Rab27a 3'UTR) or the site-directed mutant Rab27a 3'UTR (Mut Rab27a 3'UTR); B: Luciferase reporter assays in BMMs, with co-transfection of WT or Mut Rab27a 3'-UTR and miR-124 as indicated. MiR-124 mimics transfection markedly weakened luciferase activity of WT-Rab27a 3'-UTR ('Compared with Empty group, p<0.05); C: Inhibition or overexpression of miR-124 in BMMs did not alter Rab27a mRNA level; D: overexpression of miR-124 significantly inhibited Rab27a protein expression $\left({ }^{*}\right.$ Compared with control group, $\left.\mathrm{p}<0.05\right)$.

Then, we discovered that the 3'-UTR of Rab27a has a direct miR-124 binding site (Fig. 5A). To analyze whether miR-124 could inhibit expression of Rab27a by binding to its $3^{\prime}$-UTR, BMMs were cotransfected with the Rab27a $3^{\prime}$-UTR luciferase reporter vector along with a vector expressing miR-124. Then we mutated the target region in Rab27a which miR-124 binding and conducted the dual-luciferase reporter assay to evaluate whether the regulation was via targeting the predicted binding site in Rab27a was identified by miR-124 rather than other non-specific behaviors (Fig. 5A). As predicted, the luciferase activity of wildtype (WT) group exhibits remarkable inhibition by miR-124 $(p<0.05)$, however, the mutant Rab27a group showed no significant difference (Fig. 5B). To study the influence of miR-124 on mRNA and protein levels of Rab27a, qRT-PCR and western blot were conducted. Results demonstrated that inhibition of miR-124 in BMMs did not alter Rab27a mRNA level but significantly increased Rab27a protein expression (Fig. 5C and D) $(p<0.05)$. 


\section{Discussion}

Osteoporosis is a common disease which characterized by a systemic damage of bone mass and increased fracture risk, has become a major public health problem and received considerable critical attention [17-19]. A strong relationship between miRNA and pathophysiological processes has been reported in the literature [20,21]. As mentioned in the literature review, miR-124 is frequently expressed in multiple tissues to regulate different pathophysiologic processes, for instance, the development of cancer [22-26], as a new therapeutic approach to Parkinson's disease [27], promotes the pathogenesis of Crohn's Disease [28] and contributes to cholangiocyte proliferation in the cholestatic liver [29].

In recent years, the relationship between miR-124 and bone resorption has been widely investigated. For example, Lee et al. discovered that miR-124 inhibits osteoclastogenesis via NFATc [30, 31]. However, much uncertainty still exists about the relation between miR-124 and osteoclastogenesis.

In this current study, we first found that miR-124 expressed a lower level in BMMs of OVX mice. Then experiments in vitro also showed that expression level of miR-124 in BMMS was down-regulated after induction with RANKL and M-CSF. Then we found that miR-124 had no remarkable effects on BMMs proliferation. Thus, we hypothesized that miR-124 might exhibit its function via regulating osteoclast differentiation. We used miR-124 inhibitors and mimics to downregulating or upregulating miR-124 expression. As we expected, overexpression of miR-124 attenuated osteoclast differentiation in BMMs, whereas inhibition of miR-124 had an opposite effect. These results suggested that miR-124 was a negative regulator in BMMS osteoclast differentiation. Next, we tried to search for the target of miR-124 in this process. Among the candidates, we found that Rab27a might be a potential target gene for miR-124.

Previous studies have reported that Rab27a was correlated with many diseases development and advances. For example, Rab27A was found to promote tumor progression [32]; lack of Rab27A caused islet dysfunction in diabetes [33] and involved in secretion from lysosomal organelles [34].

In a previous report, the researchers found that Rab27a-deficient osteoclasts showed a markedly impaired bone resorption activity, indicating the important role of Rab27a in maintaining the normal functions of osteoclasts $[35,36]$. They also showed that depletion of Rab27a results in abnormal phenotypes in osteoclasts. In the present study, using luciferase report assay, we demonstrated that Rab27a was indeed a direct target of miR-124. Thus, we can made an explanation why overexpression of miR-124 attenuated osteoclastogenesis of BMMs. In fact, overexpression of miR-124 leaded to a markedly decrease of Rab27a, thus impairing the normal functions of osteoclasts. All these results suggested that miR124 inhibited osteoclastogenesis via suppressing the expression of Rab27a (Fig. 6).

In conclusion, our study first demonstrated that miR-124 inhibited osteoclastogenic differentiation of BMMs by suppressing Rab27a expression. What's more, the results revealed that overexpression of miR124 could inhibit osteoclastogenic

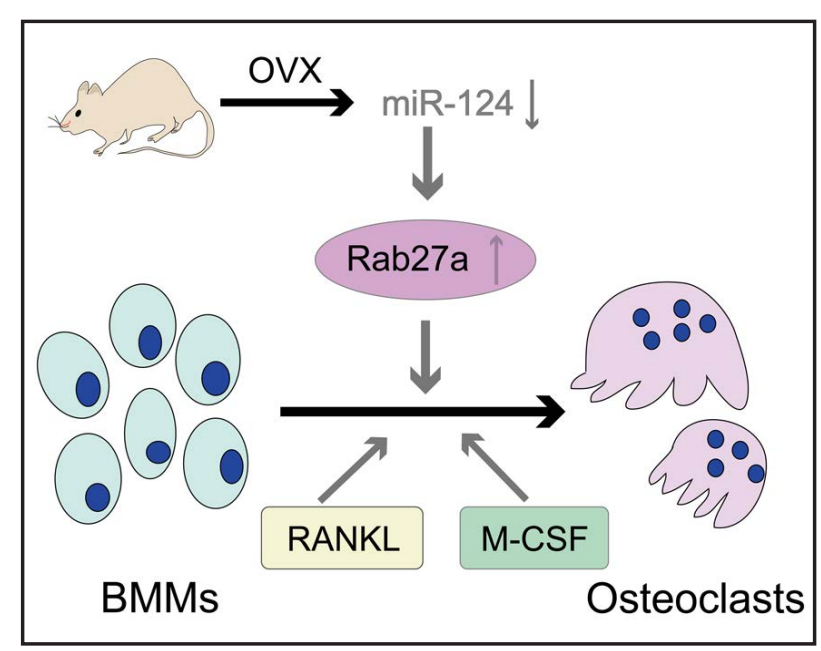

Fig. 6. Illustration for miR-124-mediated suppression of osteoclastogenesis. Reduced miR-124 in BMMs of OVX mice elevated Rab27a protein expression, thus enhanced the effects of RANKL/M-CSF-induced osteoclastogenic differentiation from BMMs to multinucleated giant osteoclasts. 


\section{Cellular Physiology Cell Physiol Biochem 2017;43:1663-1672

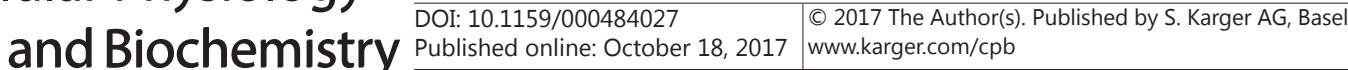

Yin et al.: Role of MiR-124 in Osteoclastogenesis

differentiation of BMMs, indicating that miR-124 might be a potential therapeutic target for the treatment of osteoporosis.

\section{Disclosure Statement}

The authors declare no conflict of interest.

\section{References}

1 Muruganandan S, Dranse HJ, Rourke JL, McMullen NM, Sinal CJ: Chemerin neutralization blocks hematopoietic stem cell osteoclastogenesis. Stem Cells 2013;31:2172-2182.

-2 Li KC, Chang YH, Yeh CL, Hu YC: Healing of osteoporotic bone defects by baculovirus-engineered bone marrow-derived mscs expressing microrna sponges. Biomaterials 2016;74:155-166.

3 Fabian MR, Sonenberg N, Filipowicz W: Regulation of mrna translation and stability by micrornas. Annu Rev Biochem 2010;79:351-379.

4 Bartel DP: Micrornas: target recognition and regulatory functions. Cell 2009;136:215-233.

5 Fabian MR, Sonenberg N: The mechanics of mirna-mediated gene silencing: a look under the hood of mirisc. Nat Struct Mol Biol 2012;19:586-593.

-6 Wan SM, Yi SK, Zhong J, Nie CH, Guan NN, Zhang WZ, Gao ZX: Dynamic mrna and mirna expression analysis in response to intermuscular bone development of blunt snout bream (megalobrama amblycephala). Sci Rep 2016;6:31050.

7 Yang L, Ge D, Cao X, Ge Y, Chen H, Wang W, Zhang H: Mir-214 attenuates osteogenic differentiation of mesenchymal stem cells via targeting fgfr1 Cell Physiol Biochem 2016;38:809-820.

-8 Gamez B, Rodriguez-Carballo E, Ventura F: Micrornas and post-transcriptional regulation of skeletal development. J Mol Endocrinol 2014;52:R179-R197.

-9 Kocijan R, Muschitz C, Geiger E, Skalicky S, Baierl A, Dormann R, Plachel F, Feichtinger X, Heimel P, Fahrleitner-Pammer A, Grillari J, Redl H, Resch H, Hackl M: Circulating microrna signatures in patients with idiopathic and postmenopausal osteoporosis and fragility fractures. J Clin Endocrinol Metab 2016;101:4125-4134.

10 Ray K: Bone: micromanaging osteoporosis. Nat Rev Rheumatol 2014;10:445.

11 An JH, Ohn JH, Song JA, Yang JY, Park H, Choi HJ, Kim SW, Kim SY, Park WY, Shin CS: Changes of microrna profile and microrna-mrna regulatory network in bones of ovariectomized mice. J Bone Miner Res 2014;29:644-656.

12 Krzeszinski JY, Wei W, Huynh H, Jin Z, Wang X, Chang TC, Xie XJ, He L, Mangala LS, Lopez-Berestein G, Sood AK, Mendell JT, Wan Y: Mir-34a blocks osteoporosis and bone metastasis by inhibiting osteoclastogenesis and tgif2. Nature 2014;512:431-435.

13 Cheng P, Chen C, He HB, Hu R, Zhou HD, Xie H, Zhu W, Dai RC, Wu XP, Liao EY, Luo XH: Mir-148a regulates osteoclastogenesis by targeting v-maf musculoaponeurotic fibrosarcoma oncogene homolog b. J Bone Miner Res 2013;28:1180-1190.

14 Fordham JB, Guilfoyle K, Naqvi AR, Nares S: Mir-142-3p is a rankl-dependent inducer of cell death in osteoclasts. Sci Rep 2016;6:24980.

-15 Lee NK, Choi YG, Baik JY, Han SY, Jeong DW, Bae YS, Kim N, Lee SY: A crucial role for reactive oxygen species in rankl-induced osteoclast differentiation. Blood 2005;106:852-859.

-16 Nishimura K, Shindo S, Movila A, Kayal R, Abdullah A, Savitri IJ, Ikeda A, Yamaguchi T, Howait M, Al-Dharrab A, Mira A, Han X, Kawai T: Trap-positive osteoclast precursors mediate ros/no-dependent bactericidal activity via tlr4. Free Radic Biol Med 2016;97:330-341.

17 Yang XC, Deng ZH, Wen T, Luo W, Xiao WF, Zhao RB, Li YS: Network Meta-Analysis of pharmacological agents for osteoporosis treatment and fracture prevention. Cell Physiol Biochem 2016;40:781-795.

18 Rachner TD, Khosla S, Hofbauer LC: Osteoporosis: now and the future. Lancet 2011;377:1276-1287.

19 Jiang T, Zhou B, Huang L, Wu H, Huang J, Liang T, Liu H, Zheng L, Zhao J: Andrographolide exerts ProOsteogenic effect by activation of Wnt/beta-Catenin signaling pathway in vitro. Cell Physiol Biochem 2015;36:2327-2339. 


\section{Cellular Physiology Cell Physiol Biochem 2017;43:1663-1672

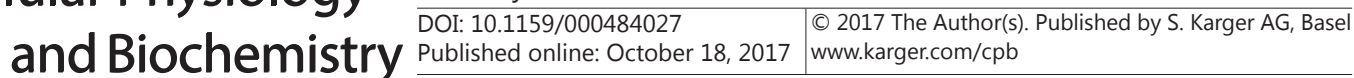 \\ Yin et al.: Role of MiR-124 in Osteoclastogenesis}

20 Sayed D, Abdellatif M: Micrornas in development and disease. Physiol Rev 2011;91:827-887.

21 Fabian MR, Sonenberg N: The mechanics of mirna-mediated gene silencing: a look under the hood of mirisc. Nat Struct Mol Biol 2012;19:586-593.

22 Ma T, Zhao Y, Wei K, Yao G, Pan C, Liu B, Xia Y, He Z, Qi X, Li Z, Wang J, Shao Y: Microrna-124 functions as a tumor suppressor by regulating cdh2 and epithelial-mesenchymal transition in non-small cell lung cancer. Cell Physiol Biochem 2016;38:1563-1574.

23 Zhou L, Xu Z, Ren X, Chen K, Xin S: Microrna-124 (mir-124) inhibits cell proliferation, metastasis and invasion in colorectal cancer by downregulating rho-associated protein kinase 1(rock1). Cell Physiol Biochem 2016;38:1785-1795.

24 Jiang CF, Li DM, Shi ZM, Wang L, Liu MM, Ge X, Liu X, Qian YC, Wen YY, Zhen LL, Lin J, Liu LZ, Jiang BH: Estrogen regulates mirna expression: implication of estrogen receptor and mir-124/akt2 in tumor growth and angiogenesis. Oncotarget 2016;7:36940-36955.

25 Zhang N, Huang Y, Wu F, Zhao Y, Li X, Shen P, Yang L, Luo Y, Yang L, He G: Codelivery of a mir-124 mimic and obatoclax by cholesterol-penetratin micelles simultaneously induces apoptosis and inhibits autophagic flux in breast cancer in vitro and in vivo. Mol Pharm 2016;13:2466-2483.

26 Zu L, Xue Y, Wang J, Fu Y, Wang X, Xiao G, Hao M, Sun X, Wang Y, Fu G, Wang J: The feedback loop between mir-124 and tgf-beta pathway plays a significant role in non-small cell lung cancer metastasis. Carcinogenesis 2016;37:333-343.

27 Saraiva C, Paiva J, Santos T, Ferreira L, Bernardino L: Microrna-124 loaded nanoparticles enhance brain repair in parkinson's disease. J Control Release 2016;235:291-305.

28 Zhao Y, Ma T, Chen W, Chen Y, Li M, Ren L, Chen J, Cao R, Feng Y, Zhang H, Shi R: Microrna-124 promotes intestinal inflammation by targeting aryl hydrocarbon receptor in crohn's disease. J Crohns Colitis 2016;10:703-712.

29 Xiao Y, Wang J, Yan W, Zhou Y, Chen Y, Zhou K, Wen J, Wang Y, Cai W: Dysregulated mir-124 and mir200 expression contribute to cholangiocyte proliferation in the cholestatic liver by targeting il-6/stat3 signalling. J Hepatol 2015;62:889-896.

-30 Lee Y, Kim HJ, Park CK, Kim YG, Lee HJ, Kim JY, Kim HH: Microrna-124 regulates osteoclast differentiation. Bone 2013;56:383-389.

31 Nakamachi Y, Ohnuma K, Uto K, Noguchi Y, Saegusa J, Kawano S: Microrna-124 inhibits the progression of adjuvant-induced arthritis in rats. Ann Rheum Dis 2016;75:601-608.

-32 Subramani D, Alahari SK: Integrin-mediated function of rab gtpases in cancer progression. Mol Cancer 2010;9:312.

33 Kowluru A: A lack of 'glue' misplaces rab27a to cause islet dysfunction in diabetes. J Pathol 2016;238:375377.

34 Mellanby RJ, Jeffery ND, Baines EA, Woodger N, Herrtage ME: Magnetic resonance imaging in the diagnosis of lymphoma involving the brachial plexus in a cat. Vet Radiol Ultrasound 2003;44:522-525.

-35 Shimada-Sugawara M, Sakai E, Okamoto K, Fukuda M, Izumi T, Yoshida N, Tsukuba T: Rab27a regulates transport of cell surface receptors modulating multinucleation and lysosome-related organelles in osteoclasts. Sci Rep 2015;5:9620.

-36 Kariya Y, Honma M, Hanamura A, Aoki S, Ninomiya T, Nakamichi Y, Udagawa N, Suzuki H: Rab27a and rab27b are involved in stimulation-dependent rankl release from secretory lysosomes in osteoblastic cells. J Bone Miner Res 2011;26:689-703. 